\title{
Precision tracking at high background rates with the ATLAS muon spectrometer
}

\section{Ralf Hertenberger*}

On behalf of the ATLAS Collaboration

Fakultät für Physik, Ludwig-Maximilians-Universität München

E-mail: R.Hertenbergerelmu.de

\begin{abstract}
Since start of data taking the ATLAS muon spectrometer performs according to specification. End of this decade after the luminosity upgrade of LHC by a factor of ten the increasing background rates require the replacement of the detectors in the most forward part of the muon spectrometer to ensure high quality muon triggering and tracking. Square meter sized micromegas detectors together with improved thin gap trigger detectors are suggested as replacement. Micromegas detectors are intrinsically high rate capable. A single hit spatial resolution below $40 \mu \mathrm{m}$ has been shown for $250 \mu \mathrm{m}$ anode strip pitch and perpendicular incidence of high energy muons or pions. The development of large micromegas structures and their investigation under non-perpendicular incidence or in high background environments is ongoing. Micromegas using resistive strip technology do not show ageing under large fluxes of low-energy neutrons. They have been successfully operated under HL-LHC equivalent fluences of $11 \mathrm{MeV}$ neutrons. In ATLAS most of the $30 \mathrm{~mm}$ diameter high-pressure drift-tubes will remain in place. They may be operated using a considerably faster and more linear drift-gas mixture. Such gas mixtures have been shown to be marginally sensitive to high photon fluences and not to age due to ionizing radiation.
\end{abstract}

36th International Conference on High Energy Physics

4-11 July 2012

Melbourne, Australia

${ }^{*}$ Speaker. 


\section{Introduction}

The ATLAS muon spectrometer [1] performs according to the specifications of efficiency, occupancy and spatial resolution at present LHC peak-luminosities of $7 \cdot 10^{33} \frac{1}{\mathrm{~cm}^{2} \mathrm{~s}}$. By end of this decade about ten times higher peak-luminosities are envisaged after the high luminosity upgrade of LHC to HL-LHC. The currently used detectors in the forward part of the muon spectrometer, the Small Wheel, need then to be replaced [2]. To cope with the expected huge background hit rates of up to $14 \mathrm{kHz} / \mathrm{cm}^{2}$ high rate capable detectors are needed to ensure high quality muon triggering, tracking and momentum reconstruction. The present Small Wheel, see figure 1, is made up of cathode strip chambers (CSC) in its inner part and $30 \mathrm{~mm}$ high-pressure drift tube detectors (MDT) in the outer region. The CSC detectors are subject to ageing. The $30 \mathrm{~mm}$ drift tube technology will start to lose efficiency and spatial resolution at local hit rates above $500 \mathrm{~Hz} / \mathrm{cm}^{2}$ $[3,4]$ due to space charge fluctuations initiated by slowly drifting ions in the gas. Figure 1 shows

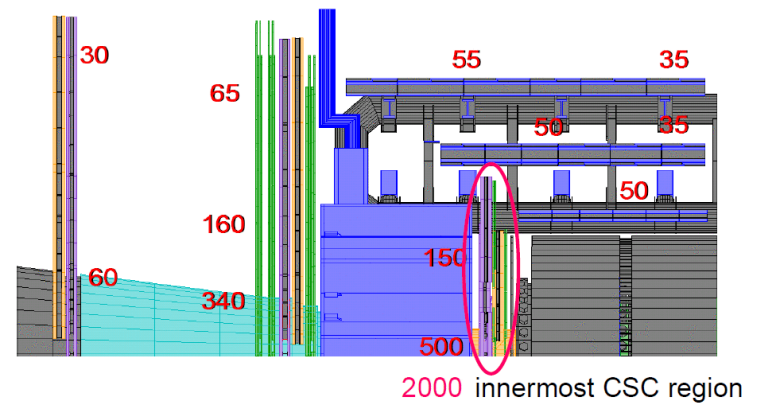

Figure 1: Simulated background induced hit-rate-densities $\left[\frac{\mathrm{Hz}}{\mathrm{cm}^{2}}\right]$ in one quarter of the muon spectrometer [5]. The Small Wheel region is encircled in red.

estimated background hit rates for the MDT chambers of one quarter of the muon spectrometer and for LHC design luminosity $L=10^{34} \frac{1}{\mathrm{~cm}^{2} \mathrm{~s}}$. At five to seven times higher luminosity background induced hit rates above $500 \mathrm{~Hz} / \mathrm{cm}^{2}$ are expected for the Small Wheel and the inner part of the first Big Wheel with up to a maximum of $14 \mathrm{kHz} / \mathrm{cm}^{2}$ in the CSC region, including a safety factor of 2. Square meter sized micromegas detectors [6] and improved thin gap trigger chambers are under discussion as replacement in the Small Wheel. Additionally, it is foreseen to improve the trigger scheme by implementing a tighter trigger association with the primary vertex. Online angular track reconstruction within $1 \mathrm{mrad}$ and a few mm position resolution is therefore required.

\section{Investigation of a large size micromegas detector with floating strip anode}

Square meter sized micromegas detectors have been suggested as replacement for the precision tracking detectors in the ATLAS Small Wheel [6]. The development of large stiff and nondeforming micromegas is ongoing. Presently, several large structures are under investigation using a $160 \mathrm{GeV}$ pion beam at CERN: a $1 \mathrm{~m}^{2}$ prototype with resistive strip anode [7] and a $0.5 \mathrm{~m} \times 0.5 \mathrm{~m}$ module with novel floating strip anode. The floating strip design is similar to the conventional micromegas design with copper made anode strips on a commercial printed circuit board. The only difference is, the strips are grounded individually by high value resistors (a few $M \Omega$ per strip) and the anode strips have to be read out capacitively. Potential discharge of the mesh after strong 
primary ionisation stops quickly, as the small sized strip will adapt the potential of the mesh by collecting a small amount of charge. As the anode consists of copper and glass-reinforced epoxy laminate, we expect no ageing.

A precise muon tracking telescope of six well aligned $9 \times 10 \mathrm{~cm}^{2}$ micromegas detectors is currently used at CERN. It allows to study uniformity, efficiency, time and position resolution at perpendicular and inclined incidence for large micromegas. These detectors have an anode strip
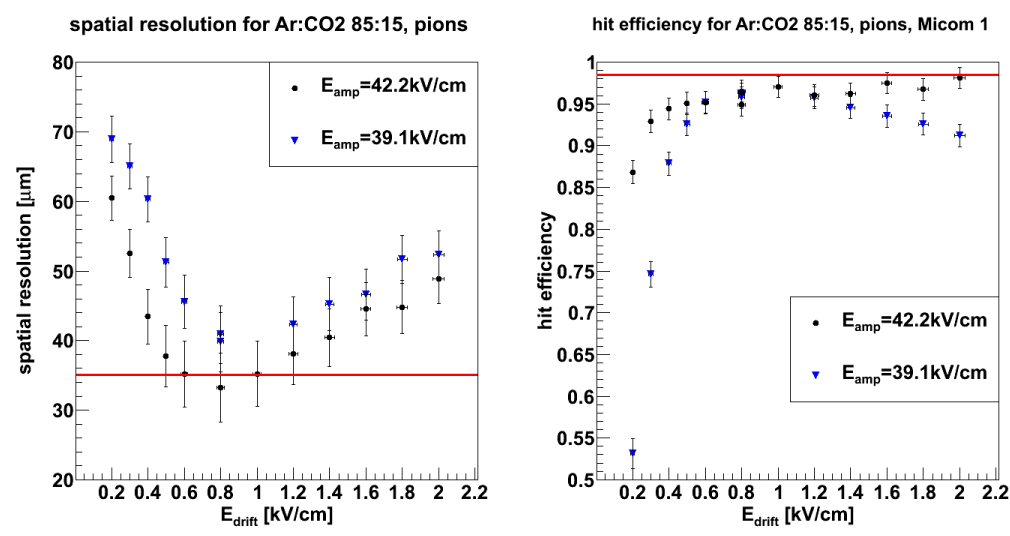

Figure 2: Left: Spatial resolution of conventional micromegas detectors with $250 \mu \mathrm{m}$ strip pitch as a function of drift-field. Right: hit efficiency as a function of drift-field, both for Ar:CO2 85:15. The inefficiency of about $1.1 \%$ is due to the insensitive area of the insulating pillars keeping the mesh $128 \mu \mathrm{m}$ above the anode. The efficiency drop above $\mathrm{E}_{\text {drift }}>1 \mathrm{kV} / \mathrm{cm}$ is due to reduced electron transparency of the mesh at $\mathrm{E}_{a m p}=39.1 \mathrm{kV} / \mathrm{cm}$

pitch of $250 \mu \mathrm{m}$, are read out by Gassiplex frontends [8] and perform according to figure 2. Single hit spatial resolution has been determined to below $35 \mu \mathrm{m}$ at high efficiency using $160 \mathrm{GeV} \pi$ and $\mu$. The setup and the method used for tracking and determination of spatial resolution is described in [9]. The signal formation in micromegas was investigated and simulated, results are discussed in detail in [8] and [10]. The combination of six micromegas detectors enables a spatial prediction of the muon interaction in the large detector with an accuracy better than $20 \mu \mathrm{m}$. The analysis of the large detector will include determination of angular resolution in single planes for inclined incidence, limited by the Landau-like clustered energy deposit of minimum ionizing particles.

\section{A Linear and fast driftgas for $\mathbf{3 0} \mathrm{mm}$ monitored drift-tube chambers}

The impact of space charge fluctuations on the spatial resolution in drift tube detectors can be strongly reduced by the use of drift gases with field-independent drift velocity of electrons and thus a linear relation of drift radius versus drift time (rt-relation). Ions need $4 \mathrm{msec}$ to reach the cathode. Induced by background they are asynchronous with the LHC clock. Muon induced electrons created at small drift radii experience then on average a decrease in electric field and thus drop in gas gain. Electrons created at large radii experience an increased electric field and thus higher drift velocity. Fluctuations lead to decrease in spatial resolution and tracking efficiency. Figure 3 shows Garfield / Magboltz [11][12] simulations of drift velocity against radius for $\mathrm{Ar}: \mathrm{CO}_{2}$ mixtures, the influence of $\mathrm{CO}_{2}$ concentration and the effect of $\mathrm{N}_{2}$ admixture. The standard ATLAS 

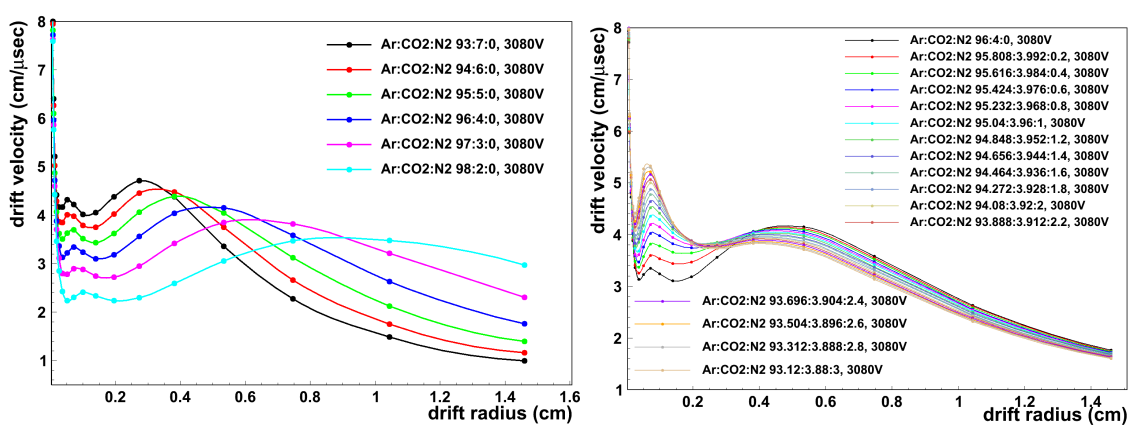

Figure 3: Garfield/Magboltz simulation: Left: $\mathrm{Ar}: \mathrm{CO}_{2}$ mixtures without $\mathrm{N}_{2}$. The amount of $\mathrm{CO}_{2}$ has been varied between 2 an 7\%. Right: The relative amount of $\mathrm{Ar}$ and $\mathrm{CO}_{2}$ was kept constant to 96:4\%. The additional amount of $\mathrm{N}_{2}$ has been varied from 0 to $2.2 \%$

MDT gas mixture is $\mathrm{Ar}: \mathrm{CO}_{2}$ 93:7\% at 3bar. Reduction of the $\mathrm{CO}_{2}$ fraction from 7 to $3 \%$ makes the drift velocity by far more homeogeneous. This was experimentally reproduced in the measurement performed at the gamma irradiation facility (GIF) at CERN, see figure 4. An occupancy reduction of about $40 \%$ is observed from the reduction in maximum drift time from 680 to $440 \mathrm{~ns}$ for the gas mixture $\mathrm{Ar}: \mathrm{CO}_{2}: \mathrm{N}_{2}$ 96:3:1, where the admixture of $1 \%$ of $\mathrm{N}_{2}$ helps for further equalization of drift velocity at radii around $1 \mathrm{~mm}$ and as additional quencher. Measurements at the test facility in Garching [13] show only slightly increased afterpulsing in comparison to $\mathrm{Ar}: \mathrm{CO}_{2}$. Comparing analog signals, the pulse duration is never longer than for Ar: $\mathrm{CO}_{2}$ 93:7 and the amplitude of the afterpulse signals is very low. Also, we did not observe any substantial amount of streamers at gas gain 20000. If at all, the total amount of charge carriers produced in the drift gas is only marginally increased. We also did not observe any substantial increase in chamber current when exposing a chamber to $1900 \mathrm{~Hz} / \mathrm{cm}^{2}$ of $662 \mathrm{keV} \gamma$ irradiation. Figure 4 summarizes the results from the GIF
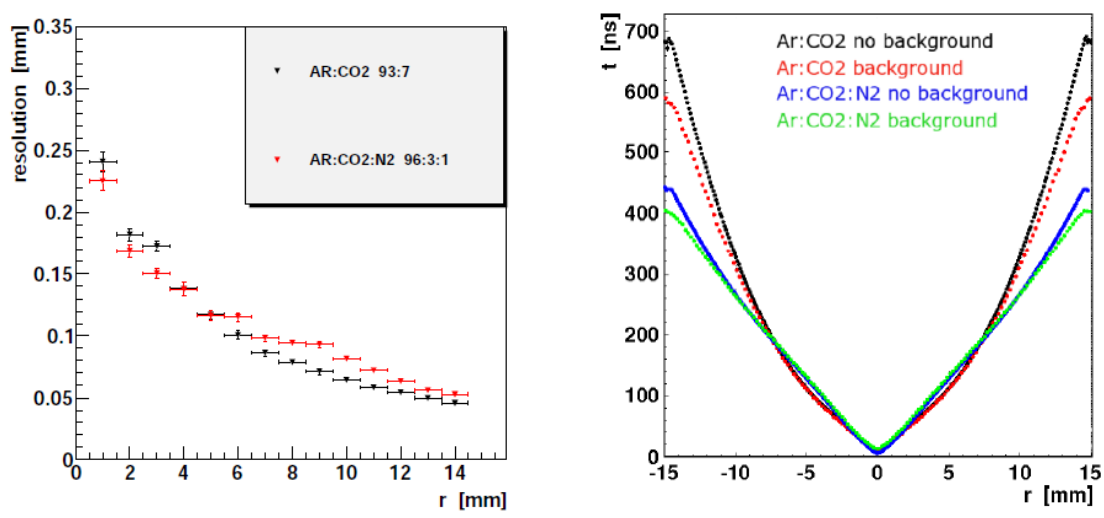

Figure 4: Measurement at the Gamma Irradiation Facility (GIF) at CERN: Left: radial dependent position resolution for both gases without background. Right: rt-realtion for both gases with and without background.

measurement. Two $50 \mathrm{~cm}$ long MDT chambers, aligned on top of each other, were sandwiched by a segmented scintillator hodoscope triggering on cosmic muons. Each chamber consisted of two multilayers, each containing three layers of six $30 \mathrm{~mm}$ drift tubes. The chambers were irradiated horizontally by $662 \mathrm{keV}{ }^{137} \mathrm{Cs} \gamma_{\mathrm{s}}$. The very top and bottom multilayer, shielded by $10 \mathrm{~cm}$ of lead, 
served as muon track reference. One of the middle multilayers was filled with standard ATLAS gas and later with the alternative drift gas. This chamber was exposed to $\gamma$ induced hit rates of up to $1900 \mathrm{~Hz} / \mathrm{cm}^{2}$. The left part of figure 4 shows the radius dependent resolution for $\mathrm{Ar}: \mathrm{CO}_{2}$ 93:7 (black points) and $\mathrm{Ar}: \mathrm{CO}_{2}: \mathrm{N}_{2}$ 96:3:1 (red points) without $\gamma$ background, no major difference is observed. Switching on the ${ }^{137} \mathrm{Cs}$ source the $\mathrm{Ar}: \mathrm{CO}_{2}: \mathrm{N}_{2}$ mixture shows considerably reduced impact on the rt-relation: a less pronounced change of averaged maximum drift time and of the averaged rt-relation. The overall position resolution integrated over all radii deteriorates only by $129 \mu \mathrm{m}$ for $\mathrm{Ar}: \mathrm{CO}_{2}: \mathrm{N}_{2}$ but by $234 \mu \mathrm{m}$ for Ar: $\mathrm{CO}_{2}$ [14]. Applying time slewing corrections, not performed in this analysis, would improve the result even further [15]. The choice of $\mathrm{Ar}: \mathrm{CO}_{2}: \mathrm{N}_{2}$ as drift gas will considerably improve the performance of $30 \mathrm{~mm}$ drift tubes at higher background rates and will reduce the occupancy by $40 \%$.

\section{Irradiation studies}

To study ageing properties, high-rate capability and the response of detectors to high energy neutrons an irradition facility has been built up at the Garching tandem laboratory. It has been used extensively for the characterisation of the $15 \mathrm{~mm}$ drift tube technology presented in [4]. Using $20 \mathrm{MeV}$ protons, we have shown that track reconstruction efficiency and spatial resolution of $15 \mathrm{~mm}$ drift-tube detectors is robust against up to $20 \mathrm{kHz} / \mathrm{cm}^{2}$ highly ionizing background hits. No sign of ageing was observed after accumulating an irradiation dose corresponding to ten years of HL-LHC operation. Details are presented in ref. [4]. Figure 5 shows the result of a similar age-

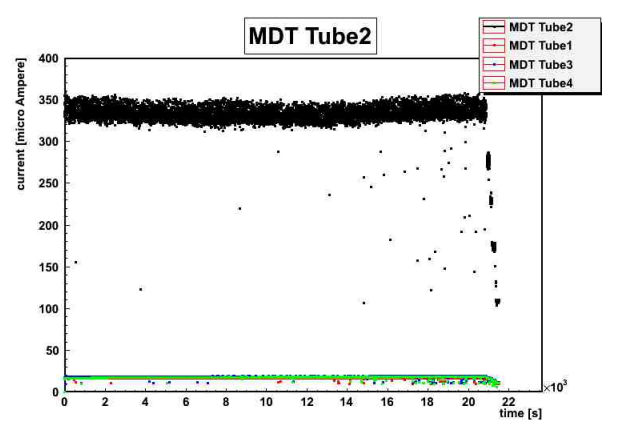

Figure 5: Current in a $30 \mathrm{~mm}$ drift tube irradiated with $105 \mathrm{nA}$ of $20 \mathrm{MeV}$ protons over $6 \mathrm{~h}$. The step function at the end of the run corresponds to the intensity of the proton beam decreased stepwise for calibration purposes.

ing study for the fast and linear drift gas $\mathrm{Ar}: \mathrm{CO}_{2}: \mathrm{N}_{2}$. The $30 \mathrm{~mm}$ drift tubes were irradiated with $105 \mathrm{nA}$ of $20 \mathrm{MeV}$ protons. The beam spot was wobbled along the tubes resulting in an irradiation region parallel to the tubes of dimension $7 \mathrm{~cm} \times 1 \mathrm{~cm}$. Within six hours of irradiation $1 \frac{\mathrm{As}}{\mathrm{cm}}$ has been accumulated, equivalent to ten years of LHC running. After irradiation the tubes were investigated using cosmic muons. Efficiency and muon response did not change comparing irradiated with non-irradiated regions of the tubes, no ageing was observed. Producing high energy neutrons $(11 \mathrm{MeV} \pm 5 \mathrm{MeV})$ the facility was used to irradiate micromegas detectors with resistive strip technology. They have been successfully operated under $11 \mathrm{MeV}$ neutron fluences of $10^{7} \frac{\text { neutrons }}{\mathrm{cm}^{2} \mathrm{~s}}$. 


\section{Summary}

A study of a $50 \mathrm{~cm} \times 50 \mathrm{~cm}$ large micromegas detector with novel floating strip anode is presently ongoing at CERN. As precision tracker we use a telescope of six $9 \mathrm{~cm} \times 10 \mathrm{~cm}$ large non-resistive micromegas with copper anode. They allow a track prediction better than $20 \mu \mathrm{m}$ at the position of the detector under test. A single hit spatial resolution below $35 \mu \mathrm{m}$ has been shown for each of these detectors with $160 \mathrm{GeV} \pi$ and $\mu$.

Ar: $\mathrm{CO}_{2}: \mathrm{N}_{2} 96: 3: 1 \%$ is suggested as fast and linear drift gas for the $30 \mathrm{~mm}$ diameter drift-tube detectors. Results from a tracking study under $1900 \mathrm{~Hz} / \mathrm{cm}^{2}$ gamma background, in agreement with simulation, show $40 \%$ less occupancy and strongly reduced sensitivity to ion space charge. The gas mixture does not age due to ionizing radiation.

In the irradiation facility at the Garching tandem laboratory micromegas using resistive strip technology have been successfully operated under $10^{7} \frac{\text { neutrons }}{\mathrm{cm}^{2} \mathrm{~s}}$ of $11 \mathrm{MeV}$.

Using the facility with $20 \mathrm{MeV}$ protons we could show that the track reconstruction efficiency and spatial resolution of $15 \mathrm{~mm}$ drift-tube detectors (sMDT) is robust against up to $20 \mathrm{kHz} / \mathrm{cm}^{2}$ highly ionizing background hits.

Acknowledgement: We acknowledge the support of BMBF.

\section{References}

[1] G. Aad et al., Eur. Phys. J. C 70 (2010) 875

[2] Letter of Intent Phase I Upgrade, CERN-LHCC-2011-012; LHCC-I-020 (2011)

[3] M. Deile et al., NIM A535 (2004) 212-215

[4] H. Kroha et al., this conference proceeding

[5] S. Baranov et al., "Estimation of Radiation Background, Impact on Detectors, Activation and Shielding Optimization in ATLAS", CERN-ATL-GEN-2005-001 (2005)

[6] MAMMA collaboration, T. Alexopoulos et al., NIM A617 no. 1-3, (2010) 161-165

[7] J. Burnens et al., NIM A640 (2011) 110-118

[8] J.Bortfeldt et al., proceedings of the 2nd int. conf. ANIMMA 2011, Ghent (2011), arXiv:1201.2062

[9] J.Bortfeldt et al., proceedings of the XII Pisa Meeting on Advanced Detectors, Elba (2012), NIM A (2012),http://dx.doi.org/10.1016/j.nima.2012.08.070, in print

[10] J. Bortfeldt, "Development of Micro-Pattern Gaseous Detectors - Micromegas", diploma thesis, LMU München, 2010, http://www.etp.physik.uni-muenchen.de/dokumente/thesis/dipl_bortfeldt.pdf

[11] Garfield, R. Veenhof, http://garfield.web.cern.ch/garfield/

[12] Magboltz, S. Biagi, http://consult.cern.ch/writeup/magboltz/

[13] N. Tyler, "Studies on Linear and Fast Drift Gases for ATLAS MDT Chambers", master thesis, LMU München, 2011, http://www.etp.physik.uni-muenchen.de/dokumente/thesis/master_ntyler.pdf

[14] A. Engl, "Precise muon drift tube detectors for high background rate conditions", PHD thesis, LMU München, 2011, http://edoc.ub.uni-muenchen.de/13361

[15] M. Deile, "Optimization and Calibration of the Drift-Tube Chambers for the ATLAS Muon Spectrometer”, PHD thesis, LMU München, 2000, http://cdsweb.cern.ch/record/624705 